\begin{tabular}{|l|l|l|}
\hline \multicolumn{3}{|c|}{ Publisherl fo } \\
\hline PublisherName & $:$ & Springer International Publishing \\
\hline PublisherLocation & $:$ & Cham \\
\hline PublisherI mprintName & $:$ & Springer \\
\hline
\end{tabular}

\title{
Automatic Image Interpolation Using Homography
}

\begin{tabular}{|c|c|c|}
\hline \multicolumn{3}{|r|}{ Articlel nfo } \\
\hline Articlel D & : & 2738 \\
\hline ArticleDOI & : & $10.1155 / 2010 / 307546$ \\
\hline ArticleCitationID & $:$ & 307546 \\
\hline ArticleSequenceNumber & : & 80 \\
\hline ArticleCategory & : & Research Article \\
\hline ArticleFirstPage & : & 1 \\
\hline ArticleLastPage & : & \\
\hline ArticleHistory & : & $\begin{array}{ll}\text { RegistrationDate } & : 2009-7-8 \\
\text { Received } & : 2009-7-8 \\
\text { Revised } & : 2010-1-25 \\
\text { Accepted } & : 2010-4-13 \\
\text { OnlineDate } & : 2010-5-20\end{array}$ \\
\hline ArticleCopyright & : & $\begin{array}{l}\text { The Author(s) } 2010 \\
\text { This article is published under license to BioMed Central Ltd. This is an open access article distributed under } \\
\text { the Creative Commons Attribution License, which permits unrestricted use, distribution, and reproduction in } \\
\text { any medium, provided the original work is properly cited. }\end{array}$ \\
\hline ArticleGrants & : & \\
\hline ArticleContext & : & 136342010201011 \\
\hline
\end{tabular}

Yi-Leh Wu, Aff1

Email: ywu@csie.ntust.edu.tw

Cheng-Yuan Tang, Aff2

Corresponding Affiliation: Aff2

Email: cytang@cc.hfu.edu.tw

Maw-Kae Hor, ${ }_{\text {Aff3 }}$

Email: hor@cs.nccu.edu.tw

Chi-Tsung Liu, Aff2

Email: m9325025@cat.hfu.edu.tw

Aff1 Department of Computer Science and Information Engineering, National Taiwan University of Science and Technology, no. 43, Section 4, Keelung Rood, Taipei 106, Taiwan,

Aff2 2Department of Information ManagementHuafan University, no. 1, Huafan Rood Shihding

TownshipTaipei 2,2301Taiwanhfu edu tw,

Department of Computer Science, National Cheng Chi University, no. 64, Section 2, ZhiNan Rood, Wenshan District, Taipei 11605, Taiwan,

While taking photographs, we often face the problem that unwanted foreground objects (e.g., vehicles, signs, and pedestrians) occlude the main subject(s). We propose to apply image interpolation (also known

and pedestrians) occlude the main subject(s). We propose to apply image interpolation (also known
as inpainting
) techniques to remove unwanted objects in the photographs and

as inpainting techniques to remove unwanted objects in the photographs and
to automatically patch the vacancy after the unwanted objects are removed. When given only a single image, if

to automatically patch the vacancy after the unwanted objects are removed. When given only a single image

the information loss after the unwanted objects in images being removed is too great, the patching results

are usually unsatisfactory. The proposed inpainting techniques employ the homographic constraints in geometry

to incorporate multiple images taken from different viewpoints. Our experiment results showed that the

proposed techniques could effectively reduce process in searching for potential patches from multiple input

images and decide the best patches for the missing regions.

\section{Introduction}

Historical sites represent the culture of a country. As a result, preserving historical sites becomes a more and more important trend in recent years. Today, the technological advancement of digital techniques can help building 3D models of the historical sites under preservation and presenting them by virtual reality. It can keep the original features of the historic sites for a long period of time. Figure 1 shows the computerized models of 


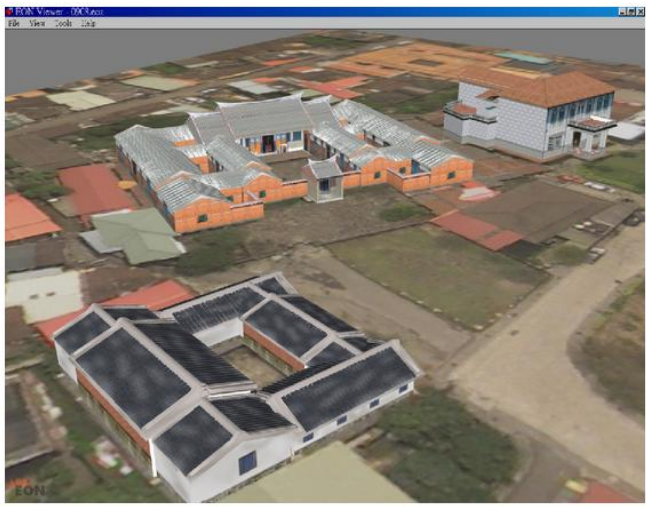

Figure 2 Virtual reality simulation walkthrough of Beipu historical sites.
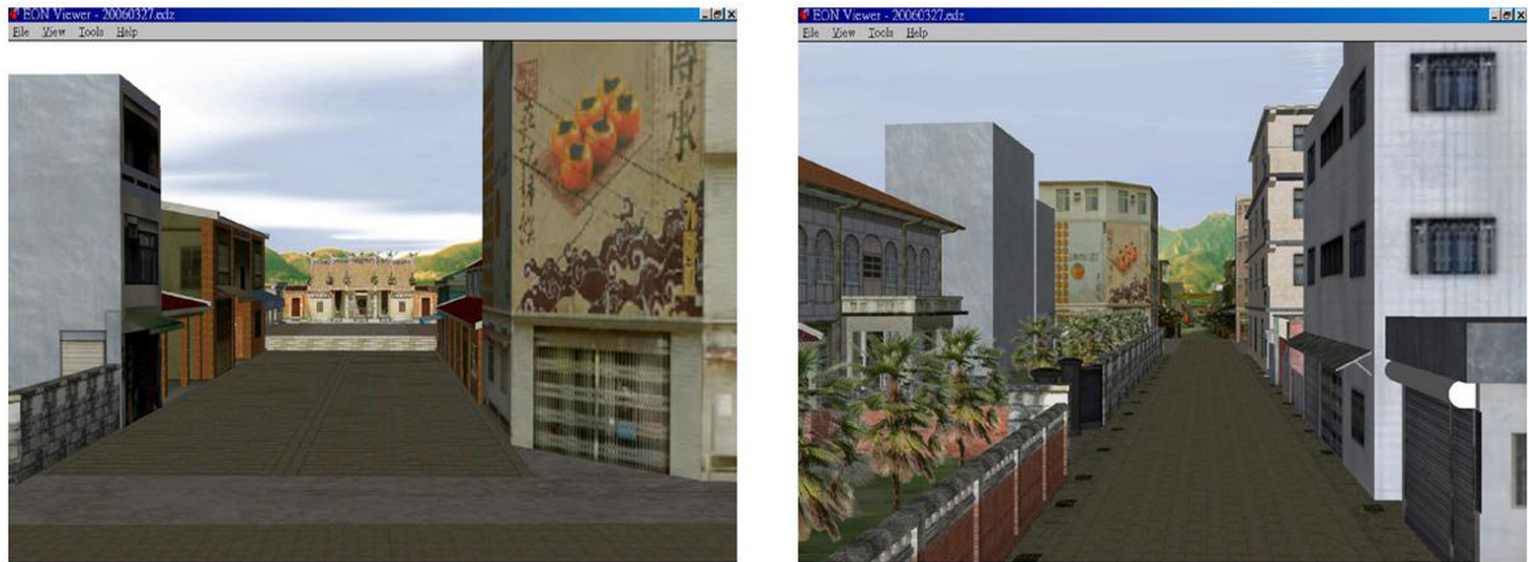

To make the 3D architecture models more visually realistic, 2D images are sometimes used as texture patches But when photographs are taken, foreground objects (e.g., tourists, vehicles, signs, etc.) sometimes occlude the main subjects. Image inpainting is the process to remove unwanted objects in the photographs and to patch the vacancy.

For example, Figure 3 is the $\mathrm{J}$ in Guang Fu historical site in Beipu Township, a traditional Hakka culture township. In Figure 3, some trees and cars occlude the main building. The usual solution is to employ image

inpainting previously proposed [1-7] to remove the unwanted trees and cars, and then patch the vacancy left behind. With image inpainting, we can correct the texture mapping problems of the reconstructed 3D models as shown in Figure 4.

\section{Figure 3 The Jin Guang Fu historical site (a) the original image, (b) the image after the} unwanted foreground objects are removed. 

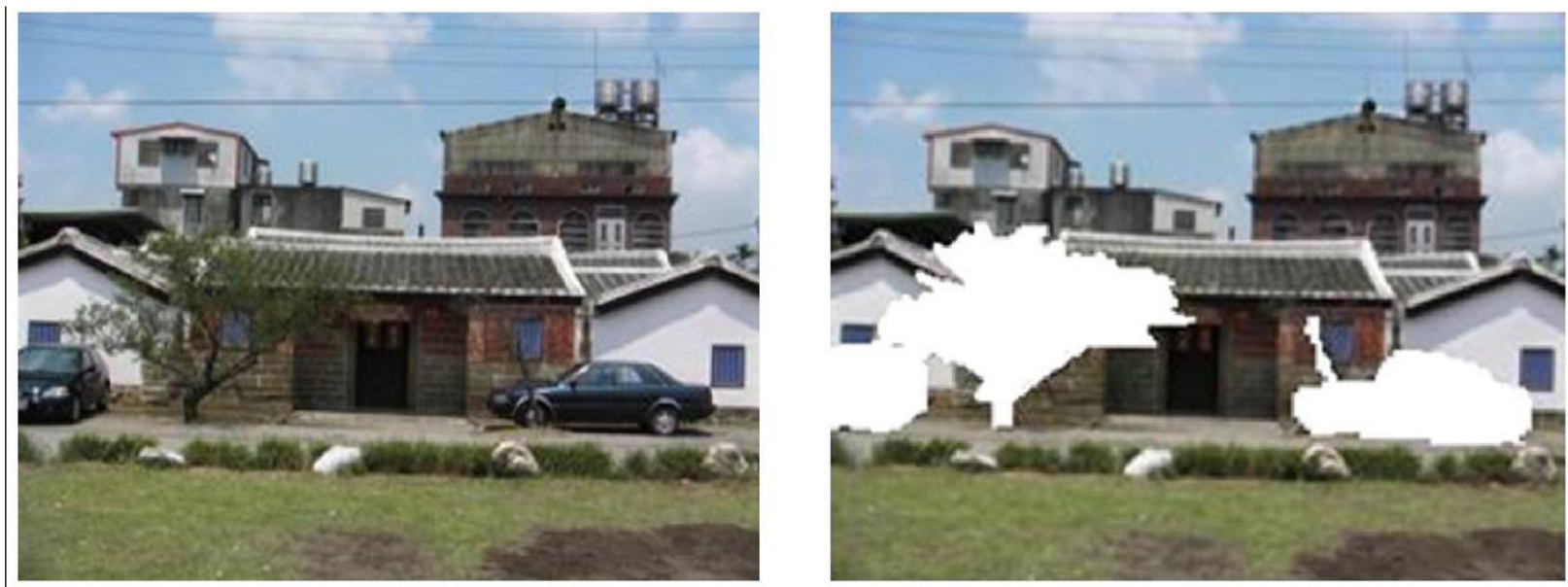

Figure 4 The J in Guang Fu historical site 3D model with texture mapping from photographs. Figure 4 The image inpainting after the foreground objects (the tree) are removed
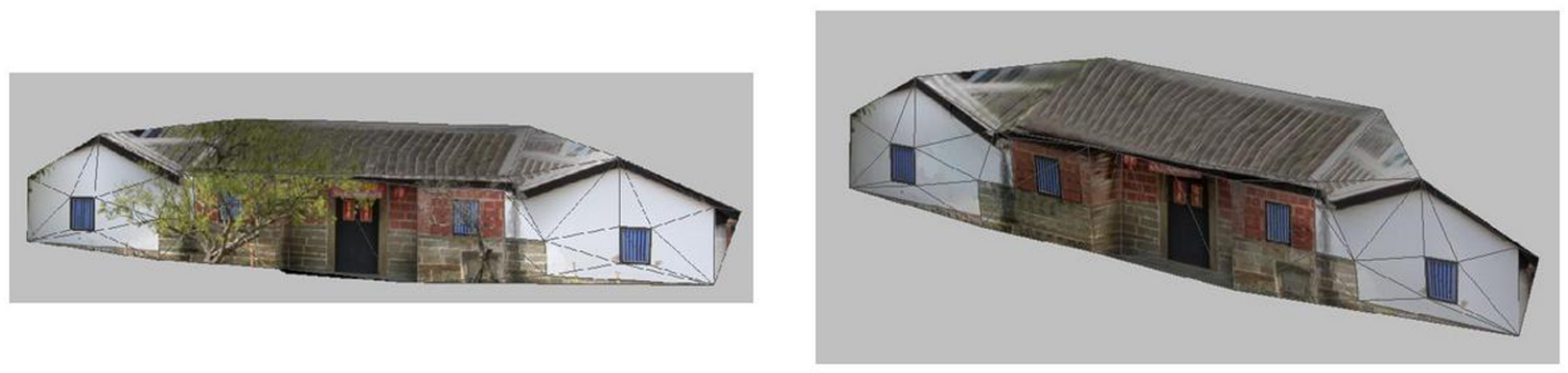

In relevant image inpainting literature, to remove damaged or undesired objects in the image, the most common method is to employ image editing tools to manually select the unwanted objects and then filled the target regions with pre-selected color. An example is shown in white in Figure 3(b). The process to patch the vacancy after unwanted objects are removed is commonly referred as image inpainting and texture synthesis in the literature [8-11].

The inpainting algorithms proposed by Oliverira et al. [1] in 2001 and the Fast Marching Method (FMM) proposed by Telea [2] in 2004 improve the speed while patching small missing or damaged regions but cause the image blurred when target regions inpainted are large.

In 2004, Criminisi et al. [3] combined the advantages of texture synthesis and image inpainting for large objects removal and inpainting. In 2005, Cheng et al. proposed a robust algorithm [4] with improved priority computation in [3]. Sun et al. (2005) proposed to first use image structure propagation and then fill the target regions [12].

In single image inpainting, the patching process relies only on the remaining image areas after undesired objects are removed. When the image information loss is too large after object removal, the patching results are usually undesirable. Some previous works also proposed the use of multiple views for inpainting [1315]. However, the method proposed in [13] relies mainly on landmarks matching without using any geometric constraints. In some cases, manual identification may still be required. In [14], the input is a series of images taken from a moving camera and motion-based background selection is employed, while our proposed method takes arbitrary views and do not reply on motion information. The proposed method in proposed method takes arbitrary views and do not reply on motion information. The proposed meth
[15] requires multiple calibrated views where our proposed method can use uncalibrated views for inpainting. Other applications of image inpainting are to restore old films and to remove and edit image objects automatically $[16,17]$

Previous research used an image for image inpainting. In this paper, we incorporate multiple images taken from different viewpoints for image inpainting. Our idea is that the regions needed for image inpainting can be correctly filled by other images taken from different viewpoints. However, incorporating multiple images taken from different viewpoints creates a challenging problem: automatic point correspondence among multiple images taken from different viewpoints is needed. In this paper, we first apply the homography property to solve the point correspondence problem among multiple images taken from different viewpoints property to solve the point correspondence problem among multiple images taken from different viewpoints for image inpainting. Based on the homography property, we used a robust method called the Least Median
of Squares (LMedS) to achieve correct point correspondences. Our main contribution is to propose an of Squares (LMedS) to achieve correct point correspondences.
automatic image interpolation algorithm for image inpainting.

\section{I mage I npainting with a Single I mage}


recover missing regions in a complex scene (composed of multiple structures and textures), such as shown

in Figure 5(a), the left windows in the images completely occluded by the tree in the foreground, from

the remaining image areas, as shown in Figure 5(b).

Figure 5 The J in Guang Fu historical site (a) the original image, (b) the image inpainting result with the structure propagation technique [12].
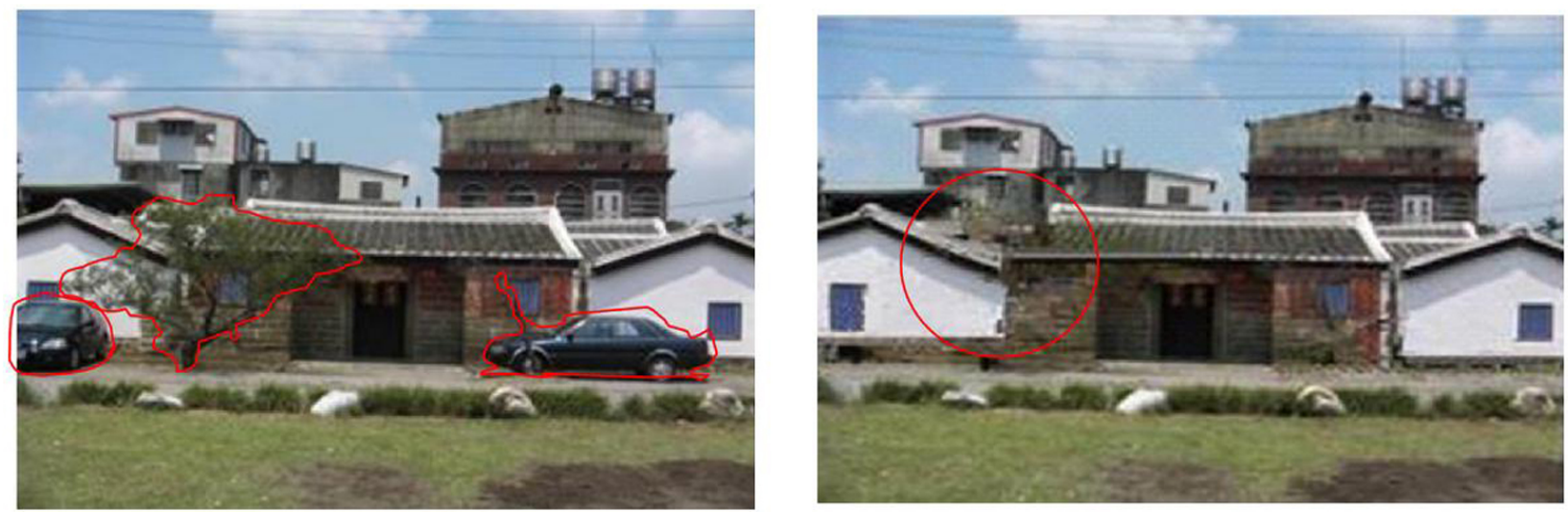

Figure 6 shows another example of the J an A historic site. When the foreground objects severely occlude the target object, the inpainting process cannot recover the actual image information of the target area after undesired objects are removed. As shown in Figure $6(\mathrm{~b})$, the image cannot be patched satisfactorily. Figure 7 shows another example of inpainting results with the exemplar-based technique, the robust priority

technique, and the structure propagation technique from a single image. Note how different techniques produce unwanted artifacts.

Figure 6 The J an A historic site, (a) the original image, (b) the image inpainting result with the exemplar-based technique [1].
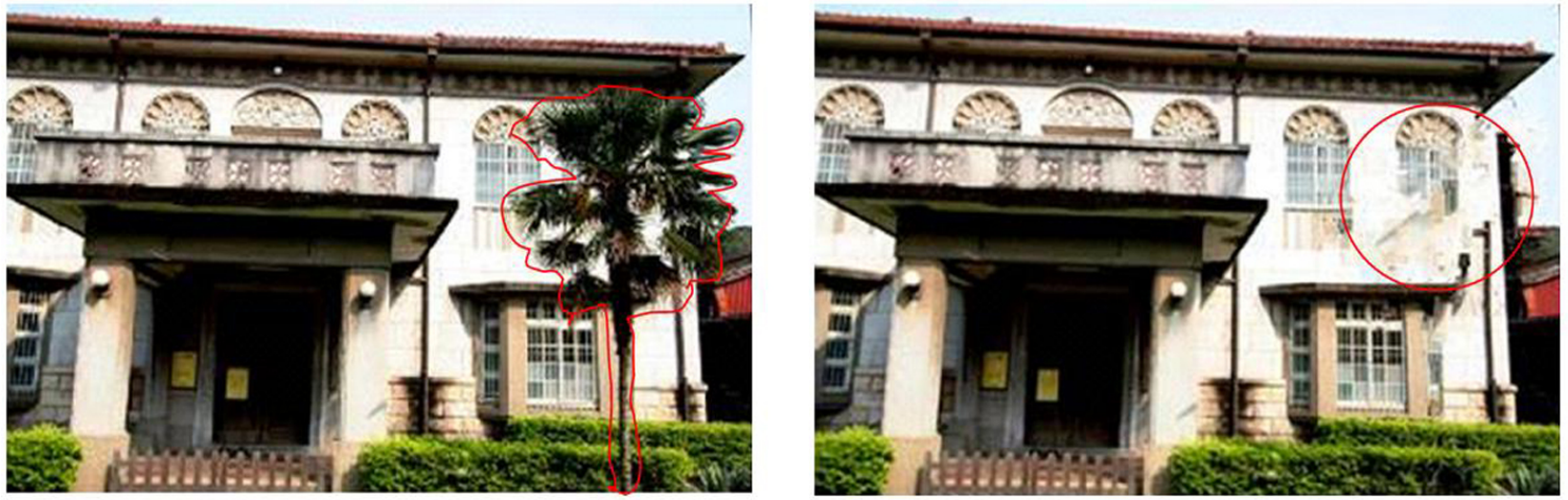

Figure 7 Different inpainting results (a) the original image, (b) the exemplar-based result, (c) the robust priority result, and (d) the structure propagation result. 

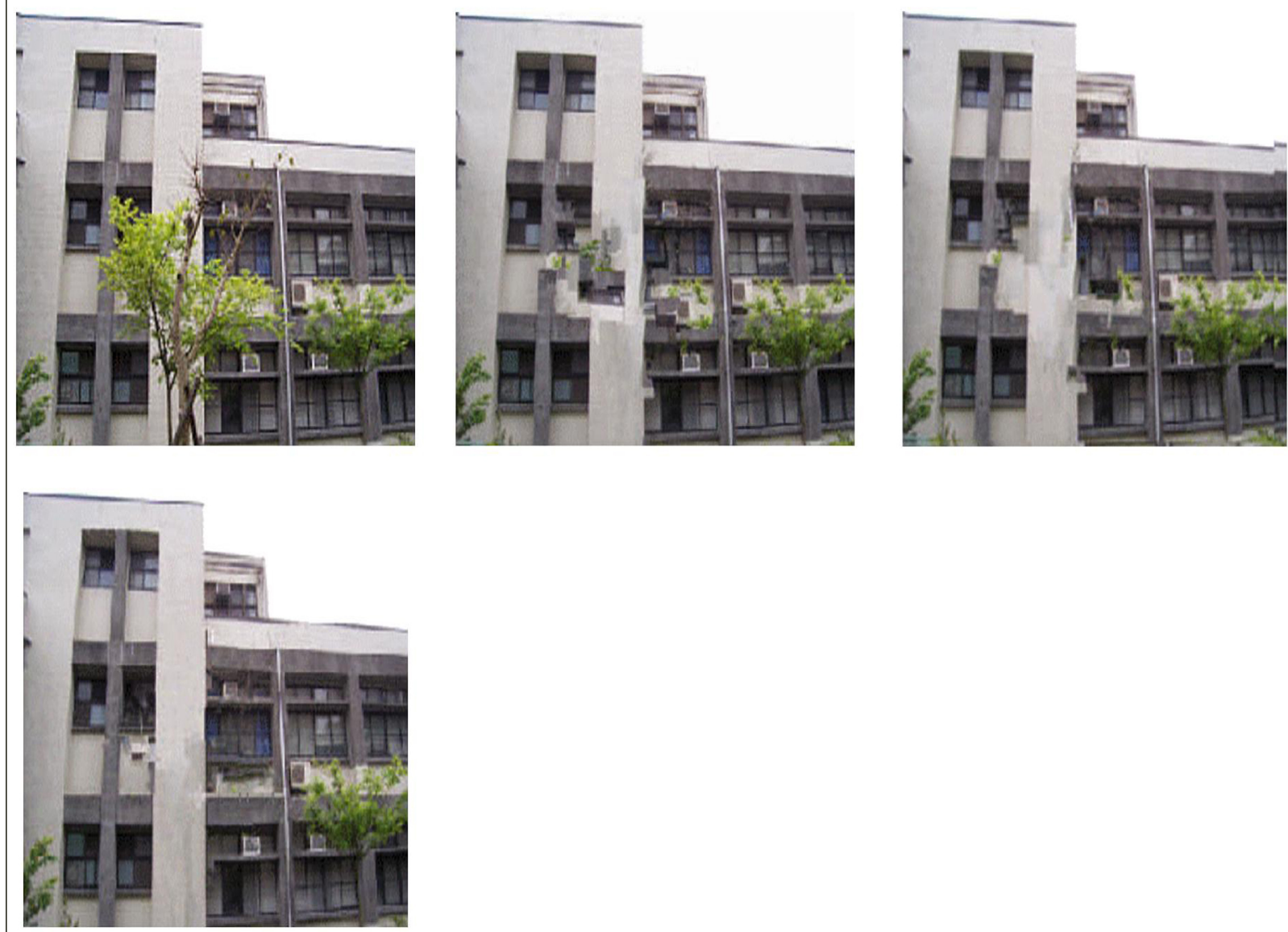

The idea of using multiple images from different shooting angles is to try to recover objects that may not be occluded in all shootings. In this following section we propose to use the homography and rectification for image inpainting with multiple images.

\section{Multiple View Geometry}

In this section we discuss the image geometric characteristic we propose to employ in image inpainting with multiple images. Projective geometry refers to the relationship among images that are formed by the projections of the light reflection of objects in $3 \mathrm{D}$ space into $2 \mathrm{D}$ images, taken by different camera rotation and translation. We then discuss in details how to employ multiple source images in image inpainting.

\subsection{Camera Geometry and Camera Model}

Projective geometry is used throughout the paper to describe the perspective projection of the scene onto the images. This projection is described as follows:

$$
\mathbf{x}=a \mathbf{P X},
$$

where $\mathbf{P}$ is $3 \times 4$ projection matrix describing the perspective projection process, $X=[x, y, z, 1]^{T}$ and $x=[x, y, 1]^{T}$ are vectors containing the homogeneous coordinates of the world points, and $d$ is a scale factor, respectively, image points.

When the ambiguity on the geometry is metric, (i.e., Euclidean up to an unknown scale factor), the camera projection matrices can be put in the following form: 


$$
\mathbf{K}=\left[\begin{array}{ccc}
f_{x} & s & u_{x} \\
& f_{y} & u_{y} \\
& & 1
\end{array}\right],
$$

where $f_{x}$ and $f_{y}$ represent the focal length divided by the horizontal and vertical pixel dimensions, $s$ is a measure of the skew, and $\left(u_{x}, u_{y}\right)$ is the principal point.

\subsection{Two-View Geometry}

Consider the image points $\mathrm{x}$ and $\mathrm{x}^{\prime}$ of a 3D point $\mathrm{X}$ observed by two cameras with optical centers $\mathrm{O}$ and $\mathrm{O}^{*}$ : These five points form a common plane, that is, defined as the so-called epipolar plane. The points $\mathbf{e}$ and $\mathrm{e}^{\prime}$ are called the epipoles of the two cameras. The epipole $\mathbf{e}^{\prime}$ is the projection of the optical center $\boldsymbol{O}$ of the first camera in the image observed by the second camera and vise versa. If $x$ and $x^{\prime}$ are images of the same point, then $\mathbf{x}^{\prime}$ must lie on the epipolar line associated with $\mathrm{X}$, that is so-called the epipolar constraint.

The epipolar constraint plays an important role in stereo vision analysis. When the internal camera parameters are known, the epipolar constraint can be represented algebraically by a $3 \times 3$ matrix, called the essential matrix. Otherwise, the epipolar constraint represented by a $3 \times 3$ matrix is called the fundamental matrix, $\mathbf{F}$

Let $x=[x, y, 1]^{T}, x^{\prime}=\left[x^{\prime}, y^{\prime}, 1\right]^{\top}$ be the image points on the first image and the second image,

respectively. Algebraically, if $x$ and $x^{\prime}$ are projected from the same 3D point $x$. Then the following equation respectively. Algebrac
should be satisfied:

$$
x^{\mathrm{T}} \mathrm{F}^{\mathrm{T}} \mathbf{x}^{\prime}=0 \text {, }
$$

where $\mathbf{F}$ is the $3 \times 3$ fundamental matrix

\subsection{Homography}

There exists a relation between the points from two images shooting from different viewing angles if the points lie on the same 3D plane. The relation could be represented as a $3 \times 3$ transformation matrix as follows, which is called the planar homography matrix (as shown in Figure 8 ):

$$
\mathbf{x}^{r}=\lambda \mathbf{H x}
$$

where $A$ is a scalar.

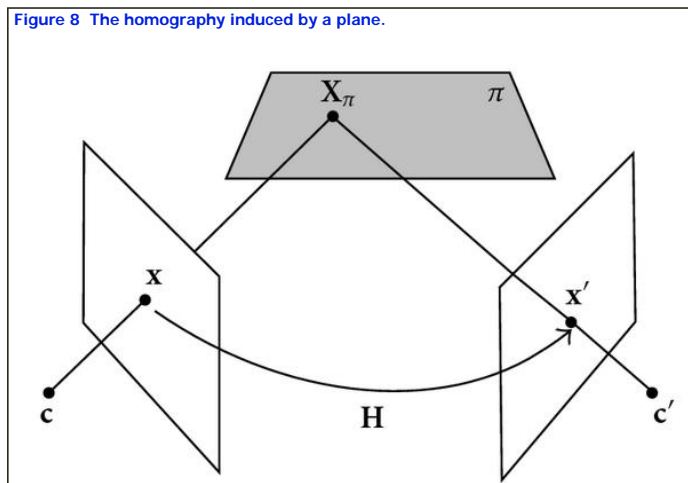

Expanding the above equation, we will derive

$$
\left[\begin{array}{c}
u^{r} \\
v^{r} \\
1
\end{array}\right]=\lambda\left\lfloor\begin{array}{lll|l}
h_{11} & h_{12} & h_{13} & u \\
h_{21} & h_{22} & h_{23} & v \\
h_{31} & h_{32} & h_{33}
\end{array}\right\rfloor
$$

From (6), we have

$$
\left[\begin{array}{l}
u^{r} \\
v^{\prime}
\end{array}\right]=\left[\begin{array}{l}
\frac{h_{11^{u}} u+h_{12} v+h_{13}}{h_{31^{u}} u+h_{32} v+h_{33}} \\
\frac{h_{21^{u}} u+h_{22} v+h_{23}}{h_{31^{u}}+h_{32} v+h_{33}}
\end{array}\right] .
$$

Equation (7) can be rewritten as

$$
\begin{aligned}
& h_{11} u+h_{12} v+h_{13}-h_{31} u u^{\prime}-h_{32} v u^{\prime}-h_{33} u^{\prime}=0, \\
& h_{21} u+h_{22} v+h_{23}-h_{31^{u}} v^{\prime}-h_{32} v v^{\prime}-h_{33^{\prime}}=0,
\end{aligned}
$$


$\left[\begin{array}{ccccccccc}u_{1} & v_{1} & 1 & 0 & 0 & 0 & -u_{1} u_{1} & -v_{1} u_{1} & -u_{1} \\ 0 & 0 & 0 & u_{1} & v_{1} & 1 & -u_{1} v_{1} & -v_{1} v_{1} & -v_{1} \\ \vdots & \vdots & \vdots & \vdots & \vdots & \vdots & \vdots & \vdots & \vdots \\ u_{n} & v_{n} & 1 & 0 & 0 & 0 & -u_{n} u_{n} & -v_{n} u_{n} & -u_{n} \\ 0 & 0 & 0 & u_{n} & v_{n} & 1 & -u_{n} v_{n} & -v_{n} v_{n} & -v_{n}\end{array}\right]_{2 n \times 9}$

$\left[\begin{array}{c}h_{11} \\ h_{12} \\ h_{13} \\ h_{21} \\ h_{22} \\ h_{23} \\ h_{31} \\ h_{32} \\ 1\end{array}\right]_{9 \times 1}=\left[\begin{array}{c}0 \\ 0 \\ \vdots \\ 0 \\ 0\end{array}\right]_{2 n \times 1}$.

If we have $n$ matched point pairs from the same 3D plane, the above equation can be used to solve the $3 \times$ 3 homography matrix $\mathbf{H}$ by applying the SVD method.

We can employ the coplanar corresponding points to determine the Homography Matrix $(\mathbf{H})$, where $\mathbf{H}$ is a $3 \times$ 3 matrix. After we decide $\mathbf{H}$, we can take the point coordinate from the first images into equation $\mathbf{x}_{i}=\mathbf{H} \mathbf{x}_{i}$ and compute the corresponding coordinate in the second image, as shown in Figure 9.
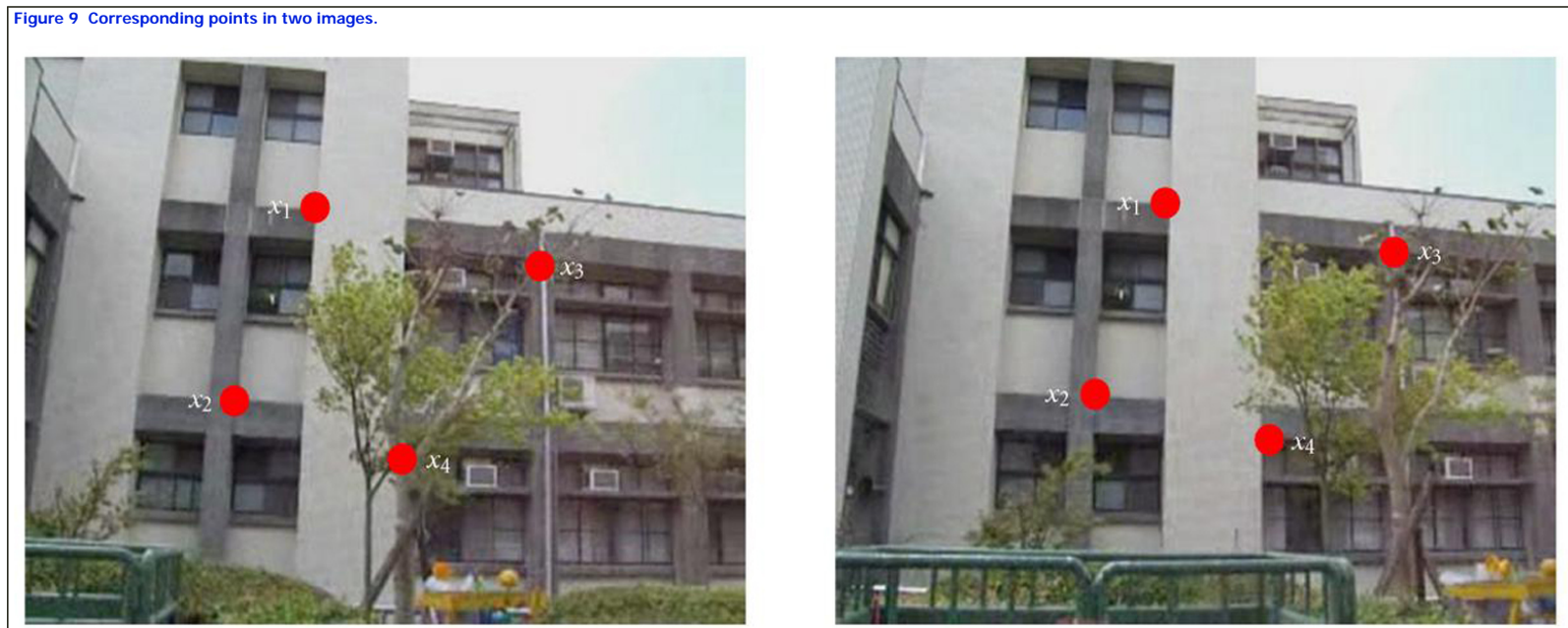

\section{Image I npainting with Multiple I mages}

In Section 2 we present the image inpainting results of different techniques with a single image. But for complex scenes, we show that after foreground removal, the information loss is too great that the missing regions cannot be recovered from the remaining image areas. The idea of using multiple images from

different shooting angles is to try to recover objects that may not be occluded in all shootings. In this section we discuss how to apply homography and rectification for image inpainting with multiple images.

Because there exist many flat surfaces where on architecture and there is a certain geometry relationship called homorgraphy, between two images, we can exploit this characteristic to locate the best fitted image patches for image inpainting.

We first take multiple images of the same objects or scenes in 3D, as depicted in Figure 10. Assuming that $X$ is a point in 3D space, since the same architecture is viewed from different angles, the corresponding point $x$ of $x$ projected in $2 \mathrm{D}$ images are at different image coordinates.

Figure 10 Multiple viewing angles in an image sequence.

[MediaObjects/13634_2009_Article_2738_Fig10_HTML.]

To calculate the homography matrix $\mathrm{H}$, one usually select four or more corresponding point sets manually. In the paper, we employ the automatic process to select potentially better corresponding point sets [18] to compute the homography matrix $\mathrm{H}$ instead of manually selecting corresponding point pairs.

First, within the selected area, we use the Sum of Squared Differences (SSD) method to locate the most likely corresponding point pairs in the two input images. The point pair with the least SSD value within there surrounding window is considered the potential corresponding point pair. SSD value within there surrounding window is considered the pote
The intermediate results of this step are as shown in Figure 12.

However, the SSD value can still produce erroneous corresponding point pairs because of conditions such as lighting and occlusion. We then employ the Least Median of Squares (LMedS) method to exclude the use of the corresponding point pairs to compute the homography matrix $\mathrm{H}$.

The LMedS is a robust estimator and can be defined as 
A random sampling strategy similar to RANSAC is adopted because the median is not differentiable. Instead of using the consensus of all $n$ data points, $m$ sample $s_{i}$ of size $k$ is randomly selected and the corresponding homography matrix $\mathrm{H}_{i}$ is computed. The residual errors $r_{i j}$ of all $r$ data points with respect to the homography matrix $\mathrm{H}_{i}$ are computed and sorted in a table as shown in Figure 13. The model $\mathrm{H}_{i}$ with the least median (minimum median residual errors) is chosen. The LMedS method can tolerate up to $50 \%$ of outliers; that is, without changing the objective function value, the LMedS method can have up to half of the data points arbitrarily far from the true estimate.

The proposed automatic process is stated as follows.

(1)Determine the features points in images with the Harris Corner Detector method (as shown in Figure 11).

(2)Select a region in the image panes. Within the selected area, use the Sum of Squared Differences (SSD) method to locate the most likely corresponding point pairs in the two input images. The intermediate results of this step are as shown in Figure 12

(3)Use the Least Median of Squares (LMedS, as shown in Figure 13) and the bucketing method (as shown in Figure 14) to determine a better corresponding point set [19].

Figure 11 Feature points located by the Harris Corner Detector method. (a) and (b) are two sample images taken from different angles and translation(s).

[MediaObjects/13634_2009_Article_2738_Fig11_HTML.]

Figure 12 Corresponding point pairs in the selected region.

[MediaObjects/13634_2009_Article_2738_Fig12_HTML.]

\section{Figure 13 Least Median of Squares method. \\ [MediaObjects/13634_2009_Article_2738_Fig13_HTML.]}

Figure 14 Bucketization for corresponding point pairs selection.

[MediaObjects/13634_2009_Article_2738_Fig14_HTML.]

Median values of all runs (as shown in Figure 13) are sorted and LMedS takes the minimum of all.

Six point pairs from different buckets are then randomly picked to compute $\mathbf{H}$. $r$ is defined as

the residual: ${ }^{r=\sqrt{\left(x_{i}-x_{i}\right)^{2}+\left(\hat{x}_{i}-x_{i}\right)^{2}}}$, where $\hat{x}_{i}, x_{i}, \hat{x}_{i}, x_{i}$ are the corresponding points in image one

and image two. Their homography geometry relationship is $\hat{x}_{i}=\mathbf{H} x_{i}, \hat{x}_{i}=\mathbf{H}^{-1} x_{i}, i=1,2,3, \ldots, m$

An example of the six selected point pairs is shown in Figure 15.

Figure 15 The six selected corresponding point pairs.

[MediaObjects/13634_2009_Article_2738_Fig15_HTML.]

With the homographic constraints, three or more images are taken from the image sequence with different shooting angles, as shown in Figure 16. The image on the top is the target image for inpainting. We compute the $\mathrm{H}$ matrix of the target image with that of the other four source images.

Figure 16 The homography matrix $\mathrm{H}$ among multiple images.

[MediaObjects/13634_2009_Article_2738_Fig16_HTML.]

The new coordinates are computed by interpolation as shown in Figure 17. The camera angles of

the source images are then transformed to be the same as the target image, as shown in Figure 18.

Figure 17 I mage transformation according to different camera angles with $\mathbf{H}$.

[MediaObjects/13634_2009_Article_2738_Fig17_HTML.]

Figure 18 I mage after projective transformation.

[MediaObjects/13634_2009_Article_2738_Fig18_HTML.]

During the inpainting process, the undesired objects were first manually removed as shown in Figure 19(b) and then the patching will be done automatically. The patching prioritization process determines the first position to be patched and compute the corresponding patches on the same position of the source images, as shown in Figure 20.

Figure 19 (a) the original image, (b) the image after object removal.

[MediaObjects/13634_2009_Article_2738_Fig19_HTML.]

Figure 20 Candidate patches from different source images.

[MediaObjects/13634_2009_Article_2738_Fig20_HTML.]

From multiple image patches, we employ SSD to compute the most similar patch and then fill the target area until the entire target region is patched. The intermediate inpainting result is shown in Figure 21.

Figure 21 Intermediate inpainting result.

[MediaObjects/13634_2009_Article_2738_Fig21_HTML.]

The complete algorithm of image inpainting with homography is detailed as follows. 
(1)Take sequential images of the objects or scenes with different shooting angles and extract the image frames, $f_{1}, f_{2}, f_{3}, \ldots, f_{n}$.

(2)Compute the homography geometry relationship among $f_{1}, f_{2}, f_{3}, \ldots, f_{n}$.

(3)Use the homography matrix $\mathrm{H}$ to transform the source images.

(4)Select manually the target region.

(5)I npaint the target region automatically:

(a)get the contour of the target region to prioritize the patching order. Compute the target patch and the source patches:

(b) use SSD to compute the similarity of the target patch and the source patches;

(c) fill the target patch with the most similar source patch.

(6) Update the target area.

(7)Repeat steps $5 \sim 6$ until the entire target area is patched.

Figure 16 shows image frames taken from multiview images of architecture with different view angles. Figure 22 shows the final inpainting result that exploits the homographic constraint to fill the target image with multiple source images. But because multiple images with different angles and brightness are used as the source images, the resulting inpainted target areas may exist slightly inconsistency in terms of brightness. But the most important image information is patched completely.

Figure 22 Inpainting with homography (a) the original image, (b) the final inpainting result. [MediaObjects/13634_2009_Article_2738_Fig22_HTML.]

Figure $\mathbf{2 3}$ depicts the J an $\mathbf{A}$ historic site from different angles as the source images for patching and Figure 24 shows the inpainting result with our proposed technique.

Figure 23 The J an A historic site from different angles.

[MediaObjects/13634_2009_Article_2738_Fig23_HTML.]

Figure 24 Inpainting result of the J an A historic site (a) the original image, (b) the image

after inpainting.

[MediaObjects/13634_2009_Article_2738_Fig24_HTML.]

Another experiment with a different set of source images of the J an A historic site is shown in

Figure 25. Figure 26 shows the inpainting result that utilizes the images from Figure 25 .

Figure 25 Another set of images of the J an A historic site from different angles.

[MediaObjects/13634_2009_Article_2738_Fig25_HTML.]

Figure 26 Another inpainting result of the J an A historic site (a) the original image, (b) the image after inpainting.

[MediaObjects/13634_2009_Article_2738_Fig26_HTML.]

\section{Conclusion}

Traditional image inpainting techniques employ a single input image. The patching process relies only on the remaining image areas after the undesired objects are removed. When the image information loss is too great after object removal, the patching results are usually undesirable.

We propose inpainting techniques that employ multiple images from different viewpoints. From multiple source images we can extract image patches that are not occluded in some images. The proposed inpainting techniques employ the homographic constraints in geometry among image frames from multiview images to assist the inpainting process. Our experiment results support that the proposed method can reduce the search process and increase the accuracy in inpainting.

Because we use multiple source images taken from different angles and under different lighting conditions, the patched area may suffer slightly inconsistency in terms of brightness. I is suggested to explore solutions to overcome this problem with increased image resolution in the future and investigate other geometric constraints that can be applied on the image inpainting process.

\begin{tabular}{|c|c|c|}
\hline \multicolumn{3}{|r|}{ BodyRef } \\
\hline FileRef & : & BodyRef/PDF/13634_2009_Article_2738.pdf \\
\hline TargetType & : & OnlinePDF \\
\hline
\end{tabular}

\section{Acknowledgments}

This work was partially supported by the National Science Council, Taiwan, under the Grant no. NSC98-2631-H-211-001 and NSC97-2221-E-011-090. 
References

1. Oliveira MM, Bowen B, McKenna R, Chang Y-S: Fast digital image inpainting. Proceedings of the International Conference on Visualization, Imaging and Image Processing(VIIP '01), 2001, Marbella, Spain

2. Telea A: An image inpainting technique based on the fast marching method. Journal of Graphics Tools 2004,9(1):25-36.

3. Criminisi A, Pérez P, Toyama K: Region filling and object removal by exemplar-based image inpainting. IEEE Transactions on Image Processing 2004,13(9):1200-1212. 10.1109/TIP.2004.833105

4. Cheng W-H, Hsieh C-W, Lin S-K, Wang C-W, Wu J-L: Robust algorithm for exemplar-based image inpainting. Proceedings of the International Conference on Computer Graphics, Imaging and Visualization, 2005

5. Drori I, Cohen-Or D, Yeshurun H: Fragment-based image completion. Proceedings of the ACM SIGGRAPH Conference on Computer Graphics, 2003

6. Cheng K-Y: Research on improving exemplar-based inpainting, M.S. thesis. Department of Computer Science and Information Engineering, National Taiwan University; 2005.

7. Levin A, Zomet A, Weiss Y: Learning how to inpaint from global image statistics. Proceedings of the 9th IEEE International Conference on Computer Vision, October 2003, Nice, France 305-312.

8. Efros AA, Leung TK: Texture synthesis by non-parametric sampling. Proceedings of the 7th IEEE International Conference on Computer Vision (ICCV'99), September 1999, Corfu, Greece 1033-1038.

9. Efros AA, Freeman WT: Image quilting for texture synthesis and transfer. Proceedings of the ACM SIGGRAPH Conference on Computer Graphics, 2001 341-346.

10. Jia J, Tang C-K: Image repairing: robust image synthesis by adaptive ND tensor voting. Proceedings of the IEEE Computer Society Conference on Computer Vision and Pattern Recognition, June 2003 643-650.

11. Tang P: Application of non-parametric texture synthesis to image, M.S. thesis. Computer Science, University of New Mexico; 2004.

12. Sun J, Yuan L, Jia J, Shum H-Y: Image completion with structure propagation. Proceedings of the ACM SIGGRAPH Conference on Computer Graphics, August 2005 861-868.

13. Kang SH, Chan TF, Soatto S: Inpainting from multiple views. Proceedings of the 1st International Symposium on $3 D$ Data Processing Visualization and Transmission (3DPVT '02), 2002

14. Rasmussen C, Korah T: Spatiotemporal inpainting for recovering texture maps of partially occluded building facades. Proceedings of the IEEE International Conference on Image Processing (ICIP '05), September 2005, Genoa, Italy 125-128.

15. Zokai S, Esteve J, Genc Y, Navab N: Multiview paraperspective projection model for diminished reality. Proceedings of the 2nd IEEE/ACM International Symposium on Mixed and Augmented Reality, 2003 217-226.

16. Patwardhan KA, Sapiro G, Benalmio M: Video inpainting of occluding and occluded objects. Proceedings of the IEEE International Conference on Image Processing (ICIP '05), September 2005, Genoa, Italy 69-72.

17. Wexler Y, Shechtman E, Irani M: Space-time video completion. Proceedings of the IEEE Computer Society Conference on Computer Vision and Pattern Recognition (CVPR '04), July 2004 1: 120-127.

18. Tang C-Y, Chou H-L, Kuo W-H, Hu P-C: Using 3D information to establish stereo correspondence from uncalibrated images. Proceedings of the 18th IPPR Conference on Computer Vision, Graphics and Image Processing, 2005, Taipei, Taiwan

19. Tang C-Y, Chou H-L, Ding Y-H: Robust methods to estimate fundamental matrices using planar constraint. Proceedings of the IEEE International Conference on Systems, Man and Cybernetics (SMC '06), 2006, Taipei, Taiwan 